\title{
Visual Deprivation Does Not Affect the Orientation and Direction Sensitivity of Relay Cells in the Lateral Geniculate Nucleus of the Cat
}

\author{
Yifeng Zhou, ${ }^{1}$ Audie G. Leventhal, ${ }^{2}$ and Kirk G. Thompson ${ }^{1, a}$ \\ 'Department of Biology, University of Science and Technology of China, Hefei, Anhui 230026, People's Republic of China \\ and ${ }^{2}$ Department of Neurobiology and Anatomy, University of Utah, School of Medicine, Salt Lake City, Utah 84103
}

\begin{abstract}
Visual deprivation in early life profoundly affects the characteristic sensitivity of visual cortical cells to stimulus orientation and direction. Recently, relay cells in the lateral geniculate nucleus (LGNd) have been shown to exhibit significant degrees of orientation and direction sensitivity. The effects of visual deprivation upon these properties of subcortical cells are unknown.

In this study cats were reared from birth to 6-12 months of age in total darkness; the orientation and direction sensitivities of area 17 (striate cortex) and LGNd cells were compared. All cells were studied using identical quantitative techniques and statistical tests designed to analyze distributions of angles. The results confirm previous work and indicate that the orientation and direction sensitivities of cells in area 17 are profoundly reduced by dark rearing. In marked contrast, these properties of LGNd relay cells are unaffected. The result is that, unlike in the normal cat, in dark-reared cats the orientation and direction sensitivities of cells in the LGNd and visual cortex do not differ.
\end{abstract}

It is concluded that (1) the orientation and direction sensitivities of cortical cells contribute little, if at all, to the sensitivities of LGNd cells since LGNd cells exhibit normal sensitivities even though the cortical cells projecting to them exhibit greatly reduced sensitivities and (2) during normal development intracortical mechanisms appear to expand upon and/or modify the weak orientation and direction sensitivities of their inputs. These intracortical mechanisms depend upon normal visual experience since in dark-reared cats, but not normal ones, the orientation and direction sensitivities of cells in the LGNd and visual cortex do not differ quantitatively or qualitatively.

[Key words: receptive field properties, stimulus selectivity, dark rearing, LGNd, area $17, X$ cell, $Y$ cell]

The effects of visual deprivation upon the receptive ficld properties of cells in the mammalian visual pathways have been studied for decades (for review see Hirsch and Leventhal, 1978; Movshon and Van Sluyters, 1981; Sherman and Spear, 1982; Jacobson, 1991). While a great deal of controversy has been

\footnotetext{
Received Apr. 12, 1994; revised June 29, 1994; accepted July 6, 1994.

This work was supported by EY04951 and EY08523 to A.G.L.

Correspondence should be addressed to Audie G. Leventhal, Department of Neurobiology and Anatomy, University of Utah School of Medicine, 50 North Medical Drive, Salt Lake City, UT 84132.

aPresent address: Department of Psychology, Vanderbilt University, Nashville, TN 37240.

Copyright (C) 1995 Society for Neuroscience $0270-6474 / 95 / 150689-10 \$ 05.00 / 0$
}

associated with this work, it is now fair to say that normal visual experience soon after birth is required for normal visual development. Notably, two of the receptive field properties of cortical neurons in cats and monkeys, the pronounced sensitivity to the angular orientation and direction of moving stimuli, have been shown to be adversely affected by procedures that eliminate exposure to oriented and directed stimuli in early life. For example, in animals reared from birth in total darkness, exposure to oriented and directed stimuli is eliminated. As a result, the number of cells in visual cortex that exhibit pronounced sensitivities to stimulus orientation and direction are reduced greatly (Blakemore and Van Sluyters, 1975; Imbert and Buisseret, 1975; Buisseret and Imbert, 1976; Leventhal and Hirsch, 1977, 1980; Fregnac and Imbert, 1978).

In the years since much of the foregoing work was done, it has been shown that many cells in the subcortical visual pathways are also sensitive to stimulus orientation and direction (Levick and Thibos, 1982; Vidyasagar and Urbas, 1982; Soodak et al., 1987; Shou and Leventhal, 1989; Smith et al., 1990; Thompson et al., 1994a). The sensitivities of cells in the dorsal lateral geniculate nucleus (LGNd) are less pronounced than are those of striate cortical cells (Levick and Thibos, 1982; Vidyasagar and Urbas, 1982; Shou and Leventhal, 1989) and are apparent in young kittens (Albus et al., 1983). In view of this work, one wonders if the profound changes in the sensitivities of cortical cells associaled with visual deprivation are associated with changes in the sensitivities of the LGNd relay cells, which both project to and receive projections from visual cortex.

The purpose of this study was to compare the orientation and direction sensitivities of cells in area 17 and in the LGNd of dark-reared cats. The results confirm previous work and show that cells in area 17 of dark-reared cats are much less sensitive to stimulus orientation and direction than are normal area 17 cells. In marked contrast, LGNd relay cells are unaffected in dark-reared animals and exhibit normal degrees of orientation and direction sensitivity. In fact, at the population level, LGNd relay cells in dark-reared animals are as selective as are area 17 cells in the same animals.

\section{Materials and Methods}

Subjects. Twenty cats reared in total darkness (Leventhal and Hirsch, 1977,1980 ) from birth to $6-12$ months of age served as subjects for this study. All cats were reared under identical conditions. Single cells were recorded from the LGNd and visual cortex. Cortical recordings provided evidence that the dark rearing was effective. Nineteen normal cats were studied for comparison.

Physiological recording procedures. Normal adult cats were prepared for electrophysiological recording as described previously (Thompson et al., 1994a). Animals were initially anesthetized with ketamine (10 
$\mathrm{mg} / \mathrm{kg}$ ) and anesthesia continued during surgery with halothane (1-2\%) in a $75 \%-25 \% \mathrm{~N}_{2} 0-\mathrm{O}_{2}$ mixture. Intravenous and tracheal cannulae were inserted. Animals were placed in a stereotaxic apparatus, and all pressure points and incisions were infiltrated with a long-acting anesthetic ( $1 \%$ lidocaine $\mathrm{HCl}$ ).

During electrophysiological recordings, a mixture of $d$-tubocurarine $(0 \mathrm{~m} / \mathrm{kg} / \mathrm{hr})$ and gallamine triethiodide $(7 \mathrm{mg} / \mathrm{kg} / \mathrm{hr})$ was infused intravenously to induce and maintain paralysis. Animals were ventilated continuously with a $75 \%-25 \% \mathrm{~N}_{2} 0-\mathrm{O}_{2}$ mixture and halothane $(0.2-1 \%)$. Body temperature was maintained at $38^{\circ} \mathrm{C}$. The ECG and EEG were monitored throughout the experiment to insure that the animal was anesthetized properly. Expired $\mathrm{pCO}_{2}$ was maintained at approximately $4 \%$.

The eyes were protected from desiccation with contact lenses. The optic disks were projected onto a tangent screen positioned $114 \mathrm{~cm}$ from the retina. These projections were determined repeatedly during the course of each recording session and were used to infer the positions of the area centrales (Fernald and Chase, 1971). Locations of the area centrales were also determined directly using the method of Pettigrew et al. (1979) to assure that their locations did not differ significantly from those inferred from the projections of the optic disks. The clarity of the optics was checked repeatedly during all experiments. Artificial pupils were used routinely. Spectacle lenses were used for correction when needed.

Receptive field mapping procedures. Visual stimuli were generated on a Tektronix (Beaverton, OR) 608 display driven by a Picasso (Cambridge, MA) image synthesizer. The Picasso was controlled by computer in conjunction with a specially designed hardware and software package developed by Cambridge Electronics (Cambridge, England). Our system is able to randomly generate a broad spectrum of visual stimuli under computer control, collect the data, and perform on-line statistical analyses. In addition, we employed an apparatus that allows an oscilloscope display to be moved to any point in the animal's visual field while at the same time maintaining a fixed distance between the display and the animal's retina. At each visual field position the center of the display screen can be $57,114,171$, or $228 \mathrm{~cm}$ from the animal's retina. In this study the screen was $57 \mathrm{~cm}$ from the retina. At this distance the mean luminance of the grating stimuli was $19 \mathrm{~cd} / \mathrm{m}^{2}$.

The responses of single cells to drifting high and low spatial frequency sinusoidal gratings as well as to stationary gratings undergoing periodic contrast reversal were used to determine whether the cell summated linearly or nonlinearly. Units were identified as X- or Y-type using the null test originally described by Enroth-Cugell and Robson (1966; and Cleland and Levick, 1974; Stone and Fukuda, 1974; Hochstein and Shapley, 1976). The spatial resolution, receptive field size, tonicity of response, response to rapid stimulus motion, and sluggishness of response were also studied.

The eccentricity of each cell's receptive field was defined as the distance from the center of the receptive field (determined by presenting stimuli to the dominant eye) to the projection of the area centralis for that eye. For all units studied, the most recent determinations of the projections of the optic disks and area centrales were used to determine eccentricity. Since receptive fields were plotted on a tangent screen, appropriate corrections were made for all receptive fields to convert receptive field size and distance from the projections of the area centrales to degrees of retinal angle. The calibrations on our optical display apparatus also provided a means of determining each unit's eccentricity and receptive field size directly.

Procedures for the determination of orientation and direction sensitivity. The physiological orientation and direction biases of LGNd and cortical cells were studied using sinusoidal gratings drifting across the receptive field (e.g., Levick and Thibos, 1982; Soodak et al., 1987) as well as drifting bars (e.g., Vidyasagar and Urbas, 1982). The orientation of each grating or bar is orthogonal to its direction of movement (the orientation is $90^{\circ}$ less than the direction). In order to better differentiate between orientation and direction sensitivity, drifting spots equal in size to the center of the cell's receptive field were also used. To generate each tuning curve, stimuli drifting in 24 or 36 different directions spaced at regular intervals from $0^{\circ}$ to $360^{\circ}$ were randomly presented to each cell. For each direction, 15-20 cycles of a drifting sinusoidal grating (temporal frequency of 2-4 Hz) or 10-15 passes of the bar or spot stimuli (velocities of $3^{\circ}-7^{\circ} / \mathrm{sec}$ ) were used to compile the tuning curves for the cells studied. The temporal frequency employed was the one judged to be optimal for the unit. The responses of the cells were studied at a variety of spatial frequencies. The various orientations and spatial fre- quencies were interleaved and presented to the cell in random order to reduce sampling bias. These procedures are like those employed by Levick and Thibos (1982) and Soodak et al. (1987) in their studies of retinal ganglion cells and LGNd cells.

When testing with sinusoidal gratings, the size of the stimulus used was at least three times larger than the receptive field center of the cell. When testing with a drifting bar, the length of the bar was at least three times the diameter of the receptive field center of the cell and the width of the har was the one judged to be optimal for the unit (generally between $0.2^{\circ}$ and $1.5^{\circ}$ ). The diameters of the drifting spots were made equal to the receptive field center of the unit being studied.

The responses of the cells to the visual stimuli presented wcre stored in the computer for later analysis. The responses to the sinusoidal gratings were defined as the amplitude of the fundamental Fourier component of the poststimulus time histogram (PSTH). For the spot and bar maps the responses were defined as the peak response of the PSTH integrated over a bin width of 150-300 msec depending on the velocity of the drifting bar or spot.

The orientation and direction preferences and sensitivities were calculated for each cell using the statistical methods described in detail in Batschelet (1981) and Zar (1974). These methods have been previously used in the calculation of the orientation sensitivities of retinal ganglion cells (Levick and Thibos, 1982; Thibos and Levick, 1985) and LGNd relay cells (Shou and Leventhal, 1989). In addition, a similar analysis has been used in the calculation of the orientation and direction sensitivities of cortical cells to moving stimuli (Wörgötter and Eyscl, 1987; Wörgötter et al., 1990). Briefly, the responses of each cell to the different directions of the stimulus presented were stored in the computer as a series of vectors. The vectors were added and divided by the sum of the absolute values of the vectors. The angle of the resultant vector gives the preferred direction of the cell. The length of the resultant vector, termed the orientation or direction bias, provides a quantitative measure of the orientation or direction sensitivity of the cell. Because the periodicity of orientation is $180^{\circ}$, the angles of the direction of the stimulus grating, bar, or spot are multiplied by a factor of two when calculating orientation preferences using the foregoing procedures. However, direction is cyclic over $360^{\circ}$, therefore the actual directions of the stimulus gratings, bars, or spots are used to calculate the direction preferences of the cell. Orientation and direction biases range from 0 to 1 with 0 being completely nonorientation sensitive or unsensitive to direction, respectively; and 1 preferring only one orientation or only one direction, respectively. Although the possible range is from 0 to 1 , the observed range of orientation biases for LGNd cells is from 0 to about 0.7 , and the range of direction biases observed for normal cat LGNd cells is from 0 to about 0.4 .

In order to provide a second measure of orientation and direction sensitivity we also calculated the maximum to minimum response ratios. In the calculation of orientation sensitivity, we averaged the responses in the preferred orientation and divided them by the averaged responses in the orthogonal orientation. Similarly, in the calculation of direction ratios we averaged the responses in the preferred direction and divided them by the averaged response in the nonpreferred direction. Theoretically, the range is from 1 to $\infty$. For the LGNd relay cells tested in this study, the observed range for orientation is from $1: 1$ to about $30: 1$ and direction is from $1: 1$ to about $6: 1$. Since the vector analysis described above takes into account the responses at all directions of stimulus motion and it can account for lopsided orientation and direction tuning (Thompson et al., 1989), it is more accurate than some previously used methods (i.e., half-width at half-height, direction index, and maximum to minimum response ratio) in predicting the orientation and direction preferences of visual neurons (Wörgötter et al., 1990).

Reliability of mapping procedures. As with orientation sensitivity, the direction sensitivity of LGNd cells in normal animals is weak compared to that of normal cortical cells. Thus we tested whether or not the preferred directions of the cells studied can be determined accurately and consistently over time. To this end we studied many cells for $1 \mathrm{hr}$ or more and compiled multiple orientation tuning curves at a variety of spatial frequencies. Using our techniques, an orientation or direction bias of 0.08 or greater indicated that the circular distribution of the cells' responses to moving stimuli was nonrandom (Rayleigh test, $p<$ 0.05 ; described below) (Zar, 1974), while an orientation or a direction bias of 0.1 or greater indicates significance at the $p<0.005$ level (Rayleigh test). Cells with biases of 0.1 or greater exhibited preferred orientations and directions within $5-10^{\circ}$ with repeated testing; their degree 

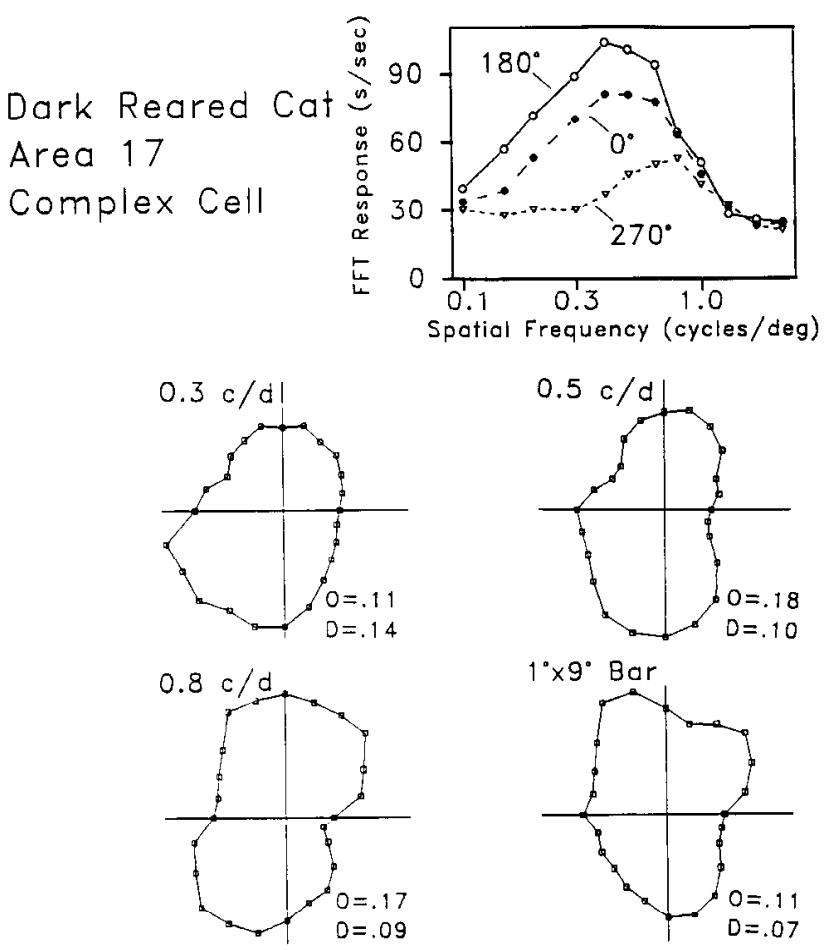

Figure 1. An example of a typical orientation- and direction-sensitive complex cell recorded from area 17 of a DR cat. The spatial frequency tuning and polar plots illustrating the orientation and direction tuning are shown. Sinusoidal gratings drifting at $2 \mathrm{~Hz}$ were used as stimuli. The spatial frequency tuning of this cell (top right) is shown for gratings drifting in three different directions $\left(0^{\circ}, 180^{\circ}\right.$, and $\left.270^{\circ}\right)$. Polar plots showing the orientation and direction tuning of this cell are shown for three different spatial frequencies. Also shown are polar plots using a drifting $1^{\circ} \times 9^{\circ}$ bar (bottom right). According to our conventions, the orientations of the drifting gratings and bars are orthogonal to the directions indicated. For example, a grating drifting in a $90^{\circ}$ or $270^{\circ}$ direction would have an orientation of $0^{\circ}$ or $180^{\circ}$. The responses to the sinusoidal gratings were defined as the amplitude of the fundamental Fourier component of the poststimulus time histogram. For spot and bar maps the responses were defined as the peak response of the poststimulus time histogram integrated over bin widths of $150 \mathrm{msec}$. Each point in the polar plots represents the response for the stimulus moving in a particular direction. The maximum response for each tuning curve was made equal to one-half the length of the axes intersecting at the center of each polar plot. All other responses were scaled to represent the percent maximum response. The responses at each spatial frequency can be inferred from the spatial frequency tuning curve (top right). For each polar plot the orientation bias $(O)$ and direction bias $(D)$ (see Materials and Methods) is shown. For spot maps (Fig. 10) the orientation bias is replaced with an axis bias (A) since a moving spot has no orientation. This cell represents the most commonly encountered type of orientation- and direction-sensitive complex cell found in area 17 of DR cats. The direction sensitivity is strongest at relatively low spatial frequencies and the orientation sensitivity is strongest at higher spatial frequencies. Also, the preferred direction is orthogonal to the preferred orientation. The orientation and direction tuning of this cell can be predicted by the spatial frequency tuning curves for the three different directions. When tested with a drifting bar, the orientation sensitivity of this cell was weaker and the direction sensitivity was very weak. Even though the strength of tuning was very weak when tested with bar stimuli, the preferred orientation and direction remained constant.

of bias varied very little between trials for a given spatial frequency. Also, in their study of retinal ganglion cell orientation bias, Thibos and Levick (1985) used an alternate method to determine significance and showed that a bias of 0.1 or greater is nearly always significant. Thus, in this study, a cell exhibiting a bias of 0.1 or greater was considered
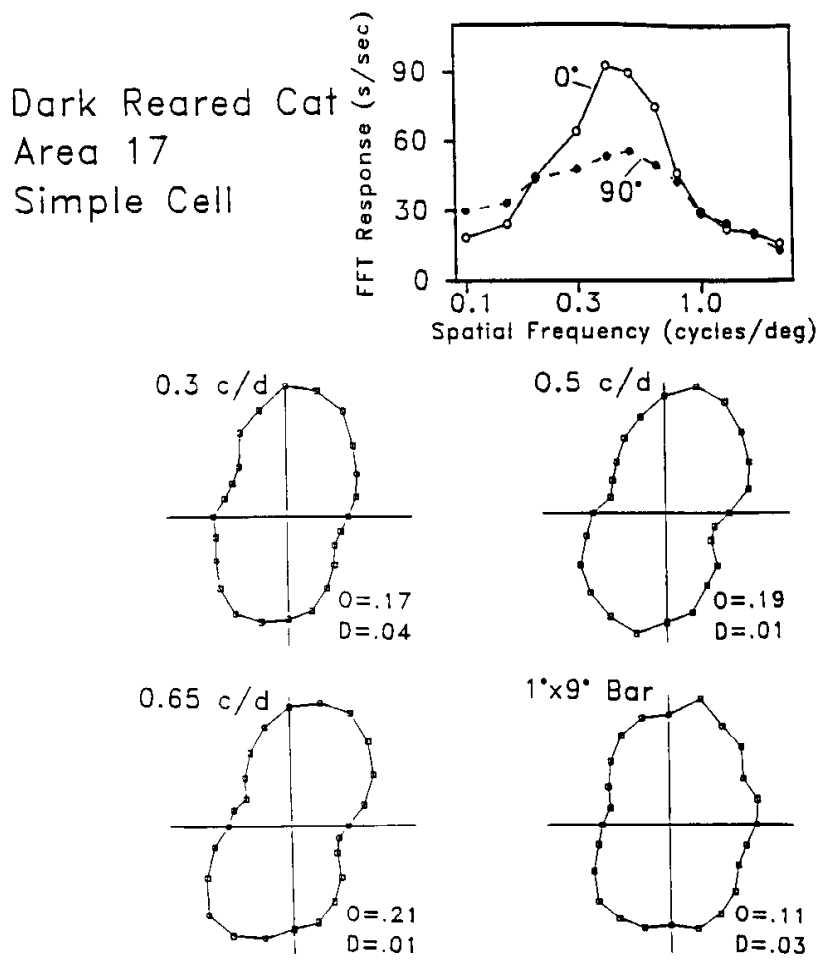

Figure 2. Polar plots and spatial frequency tuning curves for a commonly encountered type of simple cell in DR area 17. Conventions are the same as in Figure 1. Notice that the orientation sensitivity was strongest at the higher spatial frequency. Also, bar stimuli were less effective than grating stimuli in revealing the cell's selectivity.

to be orientation or direction sensitive; cells with biases less than 0.1 were defined as being unsensitive to orientation or direction. Our results indicate that a bias of 0.1 corresponds to a ratio of about $1.5: 1$.

Two other points regarding our criteria for classifying cells as selective should be made. First, the orientation tuning of the cells included in this study was determined multiple times. Random variation in the cells' responses cannot account for the orientation biases of these cells since cells were considered selective only if they exhibited the same preferred orientation with repeated testing (see above).

Second, it is unknown what degree of orientation and direction bias is functionally meaningful for visual neurons. Thus, all measures of selectivity are meaningful only in a statistical sense. In the more important functional sense, all measures of selectivity must be viewed as arbitrary until more information is obtained.

Histology and histochemistry. At the conclusion of each experiment the animal was deeply anesthetized and perfused through the heart with $700 \mathrm{ml}$ of lactated Ringer's solution containing $0.1 \%$ heparin, followed by $1000 \mathrm{ml}$ of $1 \%$ paraformaldehyde and $2.5 \%$ glutaraldehyde in $0.1 \mathrm{M}$ phosphate buffer at $\mathrm{pH} 7.4$, followed by $600 \mathrm{ml}$ of lactated Ringer's solution containing $5 \%$ dextrose. Brains were removed and the portions containing the electrode tracts were blocked and stored for $2-4 \mathrm{~d}$ in $30 \%$ sucrose solution, and then frozen sectioned at $50 \mu \mathrm{m}$. The sections were mounted on gelatinized slides, stained with thionin, and coverslipped.

Data analysis. Paired and unpaired $t$ tests as well as the Mann-Whitney $U$ test were used to compare distributions of biases. Also, several statistical techniques designed specifically to analyze distributions of angles (circular statistics) were used to help us interpret our data. A short description of each test is given below. A complete account of circular statistics can be found in Batschelet (1981).

The Rayleigh test determines if a distribution of angles differs significantly from a random distribution, that is, whether the angles are clustered about some value. If a certain angle is expected, then the $V$ test is a more powerful test of whether a distribution of angles is peaked about the expected value. To determine if the mean of the sample of angles differs significantly from an expected angle, the confidence in- 

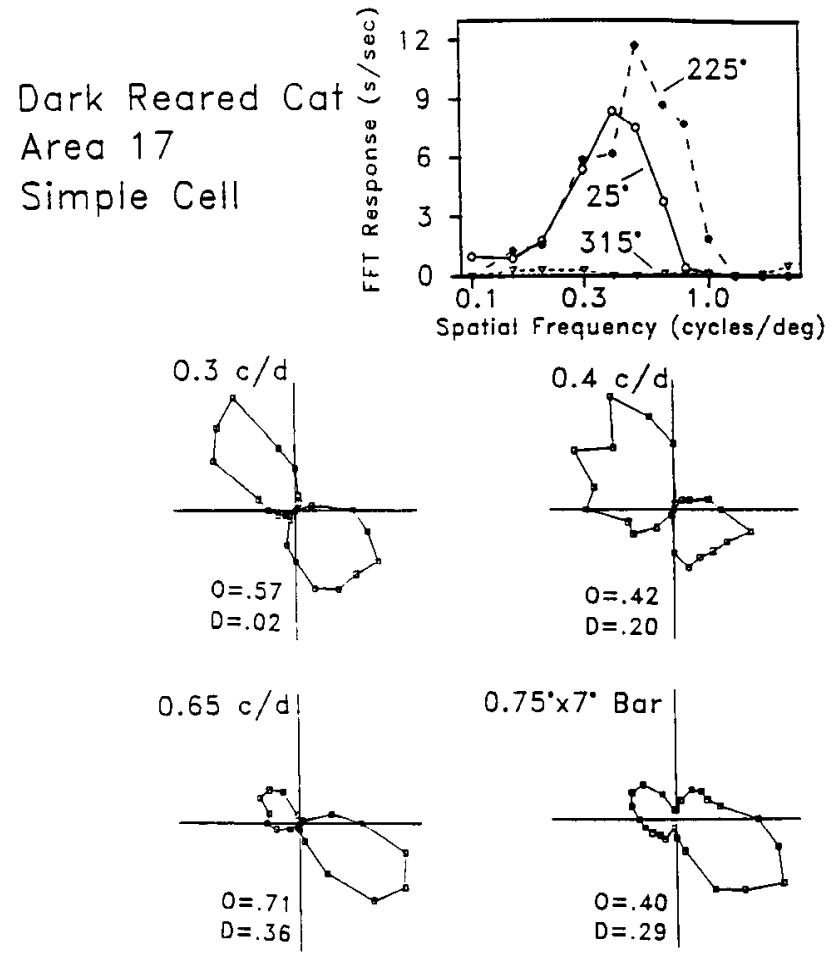

Figure 3. A rarely encountered type of orientation- and directionsensitive simple cell in DR area 17. Notice that this cell reversed its preferred direction when tested at low $(0.4 \mathrm{c} / \mathrm{d})$ and higher $(0.65 \mathrm{c} / \mathrm{d})$ spatial frequencies. Conventions are the same as in Figure 1 . This type of direction tuning has also been described for a small minority of striate cortical cells (Hammond and Pomfrett, 1989; Thompson, 1994b) and LGNd cells (Thompson 1994a) in normal cats. The spatial frequency tuning curves (top right) are consistent with the direction tuning of this cell.

tervals given by Batschelet (1981) are used. Watson's $U^{2}$ test compares two distributions of angles (unimodal or multimodal) in order to determine whether the two samples differ significantly. High $U^{2}$ values result if the two distributions are different. These techniques have been described previously (Mardia, 1972; Zar, 1974) and have been used to study anatomical and physiological orientation sensitivity in the retina (Levick and Thibos, 1982; Leventhal and Schall, 1983) and physiological orientation sensitivity in the LGNd (Shou and Leventhal, 1989).

\section{Results}

The results of the study are based upon recordings from over 1200 cells in more than 39 animals. Approximately 600 cells were studied in normal cats and 600 cells were studied in animals reared in total darkness from birth to 6-12 months of age. All cells were studied quantitatively using identical procedures. A variety of visual stimuli were employed including drifting and alternating gratings, moving and flashing bars, and spots. While the properties of cells in the LGNd and visual cortex have been studied for years, this is the first study to use identical quantitative techniques to study the effects of visual deprivation upon the selectivities of LGNd and area 17 cells in the same animals. Since it was necessary to study normal animals using the same procedures in order to collect control data, summary histograms for normal animals are presented for comparison.

\section{Orientation and direction sensitivity in the $D R$ visual cortex}

In the visual cortcx of normal cats, the large majority of cells are strongly sensitive to the orientation of moving bars and
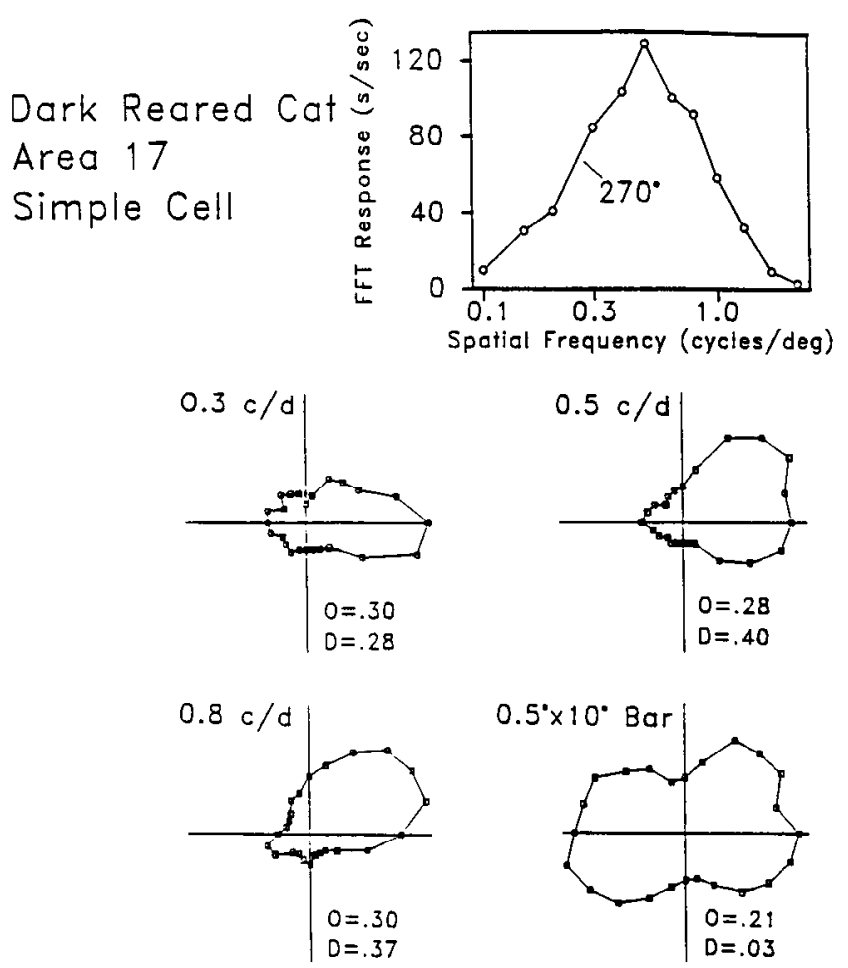

Figure 4. An area 17 simple cell that was orientation and direction sensitive at all spatial frequencies tested. This cell exhibited a weaker orientation bias and no direction biases when bars were the test stimulus. Conventions as in Figure 1. This type of cell has also been recorded from the LGNd and visual cortex of normal cats (Hammond and Pomfrett, 1989; Thompson et al., 1994 a,b).

about 35 percent are strongly sensitive to the direction of motion. Preferred orientation tends to be orthogonal to preferred direction. Simple cells tend to be more selective than complex cells and cells in layer IV tend to be more selective than cells in other layers (reviewed in Henry, 1991; Orban, 1991).

The present results confirm previous reports and show that the orientation and direction sensitivities of both simple and complex cells are greatly reduced in DR cats. Figure 1 shows the spatial frequency, orientation, and direction tuning of a complex cell recorded from the infragranular layers of area 17. Notice that the cell exhibited weak orientation and direction biases. Direction sensitivity was clearest at relativity low spatial frequencies while orientation sensitivity was clearest at higher spatial frequencies. Figure 1 also shows, as is typical of complex cells in dark-reared cats, bar stimuli were less effective then grating stimuli in revealing the cell's orientation and direction sensitivity.

Figure 2 shows the spatial frequency, orientation, and direction tuning of a typical simple cell in DR cortex. Like DR complex cells, most DR simple cells exhibit only weak orientation and direction biases, and gratings are more effective than bars at revealing the cells sensitivity.

Previous studies of DR cortex have revealed a small number of cells that exhibited relatively strong orientation and direction sensitivities; most of these cells were layer IV simple cells (Blakemore and Van Sluyters, 1975; Leventhal and Hirsch, 1977, 1980; Fregnac and Imbert, 1978). Figures 3 and 4 illustrate two simple cells. Both exhibit pronounced orientation and direction sensitivity. Like the weakly selective cells illustrated in Figures 

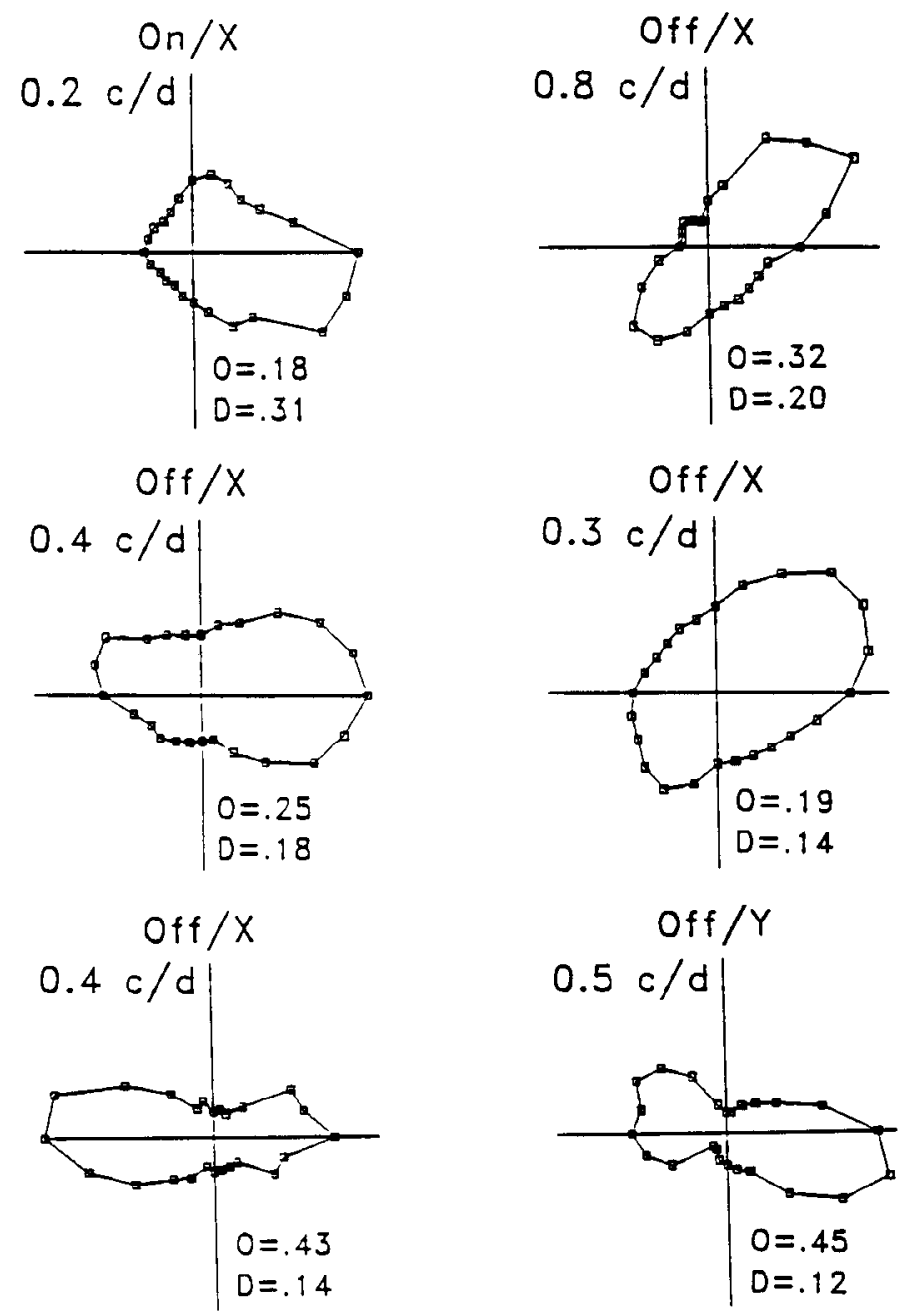

Figure 5. The orientation and direction tuning of six different DR LGNd cells that exhibited both orientation and direction sensitivity at a single spatial frequency. Notice that the preferred directions were roughly orthogonal to the preferred orientations. Conventions as in Figure 1.

1 and 2 , the selectivities of these cells were clearest when gratings of the appropriate spatial frequency, rather then bars, were the test stimuli.

The simple cell in Figure 3 exhibited a rare type of direction sensitivity observed in the LGNd and area 17 (Hammond and Pomfrett, 1989; Thompson et al., 1994a,b) of normal and deprived cats. This cell's preferred direction reversed as spatial frequency increased and the cell was most direction sensitive at a relatively high spatial frequency. The cell in Figure 4 was also atypical in that its selectivity was spatial frequency invariant. This type of cell has also been recorded from the LGNd and visual cortex of normal cats (Hammond and Pomfrett, 1989; Thompson et al., 1994a,b).

\section{Orientation and direction sensitivity in the $D R L G N d$}

The spatial frequency, orientation, and direction tuning of relay cells recorded from the A laminae of the DR LGNd are shown in Figures 5-7. The cells in Figure 5 are typical in that they exhibited clear direction biases at low spatial frequencies and clear orientation biases at higher spatial frequencies. Notice that
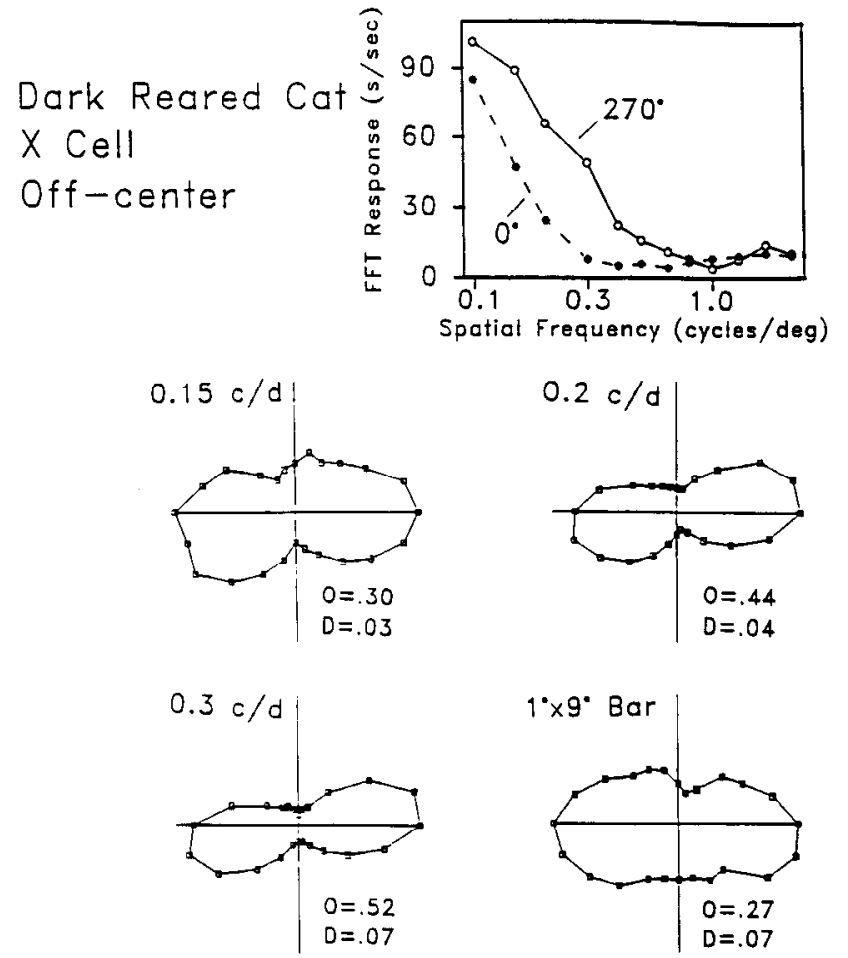

Figure 6. Polar plots and spatial frequency tuning curves for a highly selective $X$ cell recorded from the LGNd A laminae of a DR cat. This cell also illustrates a commonly encountered type of orientation tuning. Conventions are the same as in Figure 1. Notice that the orientation sensitivity was strongest at the higher spatial frequency. Bar stimuli were less effective than grating stimuli in revealing the cell's selectivity.

all cells illustrated were sensitive to both orientation and direction. Preferred orientation tended to be orthogonal to preferred direction.

The $\mathrm{X}$ cell in Figure 6 is shown because it is among the most orientation sensitive encountered in dark-reared and normal cats. It is also within the range of the most orientation-sensitive cells encountered in dark-reared cortex. As is the case for most LGNd cells, the orientation sensitivity of this cell was clearest when the test stimuli were gratings of relativity high spatial frequency and bar stimuli were less effective than grating stimuli at revealing the cells selectivity.

The DR Y cell in Figure 7 is included because it represents a relatively rare type of cell found in both the normal and DR cortex and LGNd (Thompson et al., 1994a,b). This cell is clearly sensitive to both orientation and direction. However, its direction sensitivity was clearest at relatively high spatial frequencies and its preferred direction was parallel, not perpendicular, to its preferred orientation. The Y cell shown in Figure 7 was also unusual in that its orientation selectivity was clear when bars and gratings of appropriate spatial frequency were the test stimuli. This is, of course, a common feature of cells in normal area 17 but a relatively rare feature of cells in area 17 of $\mathrm{DR}$ cats (see Fig. 9).

\section{Spatial frequency dependence of orientation and direction sensitivity in $D R$ cats}

The histogram in Figure 8 illustrates the dependence upon spatial frequency of the orientation and direction sensitivity of cells in the LGNd of DR cats. In this figure the spatial frequency 

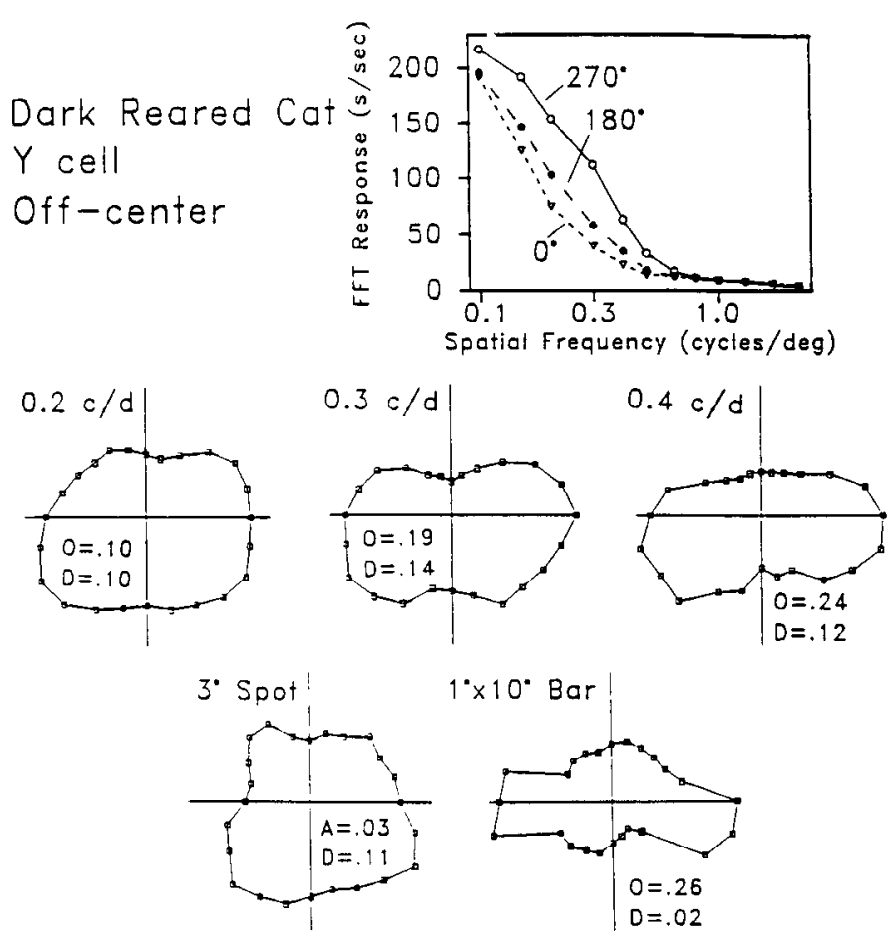

Figure 7. A rarely encountered type of orientation- and directionsensitive Y cell recorded from the LGNd of a DR cat. Conventions are the same as in Figure 1. The preferred direction of this cell was parallel, not perpendicular, to the cell's preferred orientation. This type of direction tuning has also been described for a small minority of LGNd and area 17 cells in normal cats (Thompson, 1994a,b). The spatial frequency tuning curves (top right) are consistent with the direction tuning of this cell. Notice that this cell is also atypical in that bar stimuli were as effective as grating stimuli at revealing the orientation sensitivity, but not the direction sensitivity, of the cell.

that elicited the most orientation- or direction-sensitive response was divided by the spatial frequency that elicited the strongest response from the cell. Thus, a value of one indicates that the cell was most selective at its optimal spatial frequency. It is clcar from this figurc, as the example cells showed, that orientation sensitivity is clearest at relativity high spatial frequencies while direction selectivity is clearest at relatively low spatial frequencies (Mann-Whitney $U$ test $p<0.001$ ). It should also be noted that similar spatial frequency dependencies were observed in the visual cortex of DR cats (Mann-Whitney $U$ test $p<0.001)$. The spatial frequency dependencies of cells in the visual cortex of normal cats have been described previously (Hammond and Pomfrett, 1989; Thompson et al., 1994b).

\section{A comparison of normal and dark-reared cats}

Orientation and direction sensitivity. Histograms illustrating the major results of this study are shown in Figures 9 and 10. The data for normal animals were collected using procedures identical to those used to study DR animals. Figures 9 and 10 show that for orientation and direction, respectively, the distributions of the orientation biases in the normal LGNd, DR LGNd, and DR visual cortex do not differ significantly. In all cases, gratings are more effective than bars in revealing the cells selectivity. Figures 9 and 10 also show, for orientation and direction sensitivity, respectively, that cortical cells in normal animals are much more selective than in deprived animals $(t$ test $P<$

\section{Dark Reared Cat LGNd}

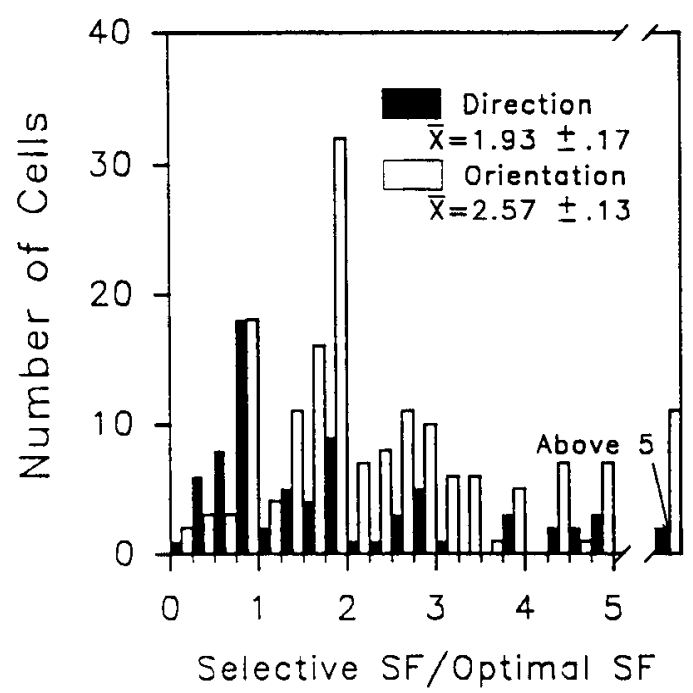

Figure 8. Histograms showing the distributions of the spatial frequency ratios of DR LGNd cells. This figure illustrates the dependence of the orientation- and direction-sensitive responses of DR LGNd cells on the spatial frequency of the test grating. A value less than 1 means that the spatial frequency which produced the most orientation- or direction-sensitive response was less than the optimal spatial frequency for the cell, while a value greater than 1 indicates that the spatial frequency which produced the most orientation- or direction-sensitive response was greater than the optimal spatial frequency for the cell. Direction sensitivity (black bars) is usually strongest at relatively low spatial frequencies, while orientation sensitivity (white bars) is usually strongest at relatively high spatial frequencies.

0.000001 ). Moreover, in normal area 17, gratings are only slightly more effective than bars in revealing the cells selectivity.

In addition to comparing all cells studied in the different groups, the mean orientation and direction biases of cells were determined for all cells recorded from the LGNd and visual cortex in each cat. When the data from individual animals were treated as individual observations, the orientation selectivities of normal and DR LGNd cells did not differ ( $t$ test $P<0.16$ ), nor did the selectivities of DR LGNd and DR cortical cells $(t$ test $P<0.6$ ). Thus, it can be concluded that no single animal or small number of animals exerted a disproportionate influence on the results.

Proportion of $X$ and $Y$ cells. It has been reported that dark rearing results in a reduction in the proportion of $Y$ cells in the LGNd (Kratz et al., 1979). In this study some $Y$ cells were found in the LGNd of DR cats. However, the proportion of cells exhibiting typical $Y$ cell properties was 14 percent in DR cats compared to 39 percent in normal animals. If the results for each cat are treated as an individual observation, this difference is significant ( $t$ test $P<0.0002$ ). The Y cells that were encountered in DR cats exhibited normal orientation and direction sensitivity.

Spatial frequency tuning. The spatial frequency tunings of LGNd cells in normal and DR cats were compared. No differences were observed in either the optimal spatial frequency or the spatial frequency limits of cells in the two groups.

Receptive field size. The mean receptive field diameter of $\mathrm{LGNd}$ cells was smaller (about 17\%) in DR cats than in normal cats ( $t$ test $P<0.05$ ). Presumably, this is due to the relatively small 


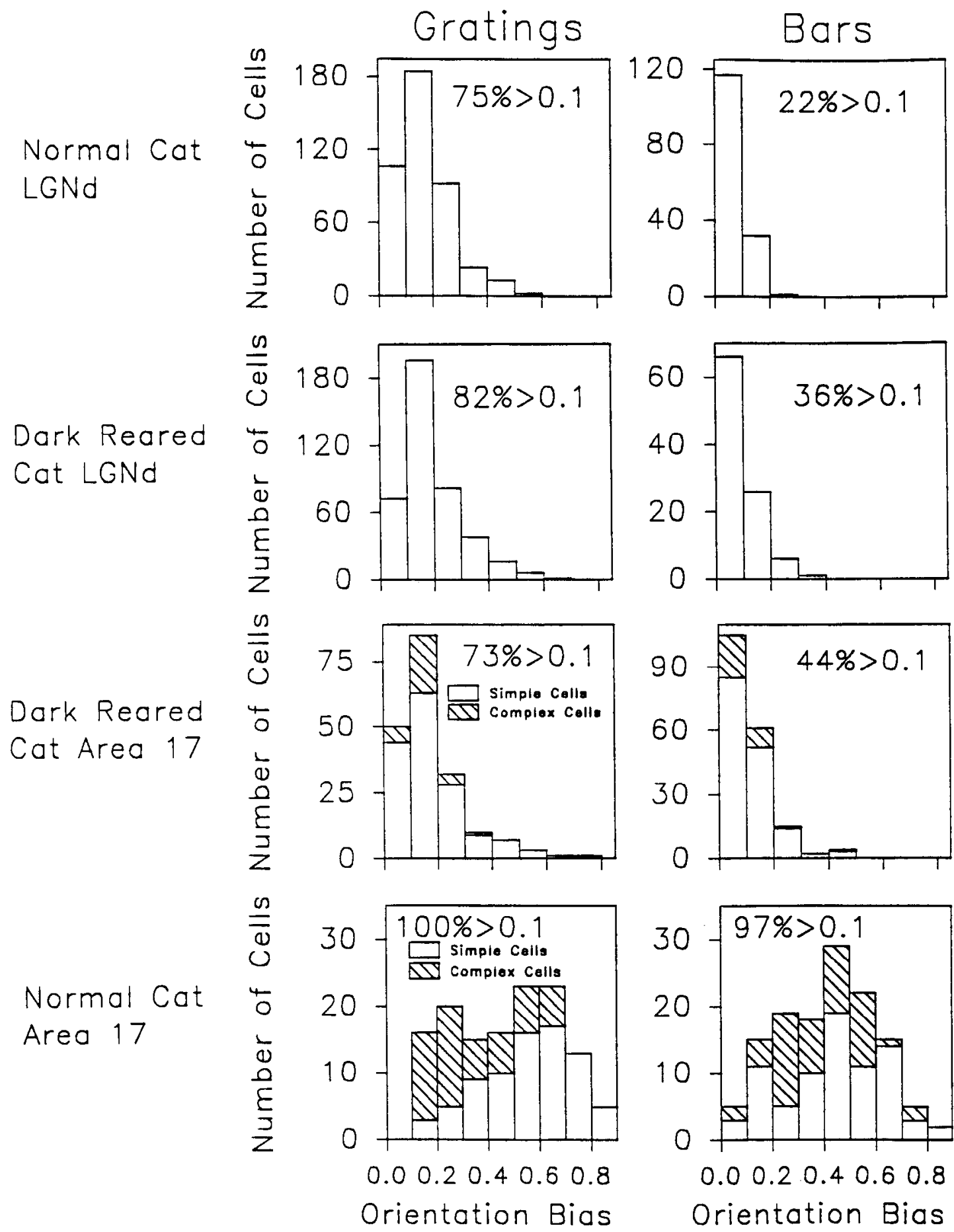

Figure 9. The distributions of the orientation biases of cells in area 17 and the LGNd of normal and DR cats. Data collected in response to drifting sinusoidal gratings and drifting bars are shown separately. The best orientation biases obtained for each cell were used to construct the histograms. A cell was considered to be significantly biased if the bias was greater than or equal to 0.1 (see Materials and Methods).

number of cells in DR cats with large receptive fields (Y cells; Cleland and Levick, 1974; Stone and Fukuda, 1974).

Maximum response. The maximum responses elicited by visual stimuli were determined for LGNd cells and area 17 cells in normal and DR cats. The results indicate that LGNd cells exhibit greater maximum responses in DR cats than normal ones ( $t$ test $P<0.00001)$. In contrast, area 17 cells exhibit greater maximum responses in normal cats than in DR cats $(t$ test $p<$ 0.01).
Spontaneous activity. The spontaneous activity of LGNd cells was greater in DR cats than normal cats ( $t$ test $P<0.05)$.

Response to flashing stimuli. The spatial distributions of on and off subregions were determined for area 17 cells in DR and normal cats. The results indicate that the proportion of area 17 cells exhibiting on/off regions was $16 \%$ in DR cats and $40 \%$ in normal cats. The proportion exhibiting distinct on or off subregions was $77 \%$ in DR cats and $60 \%$ in normal cats. In DR cats, $7 \%$ of cells could not be categorized in this fashion. 


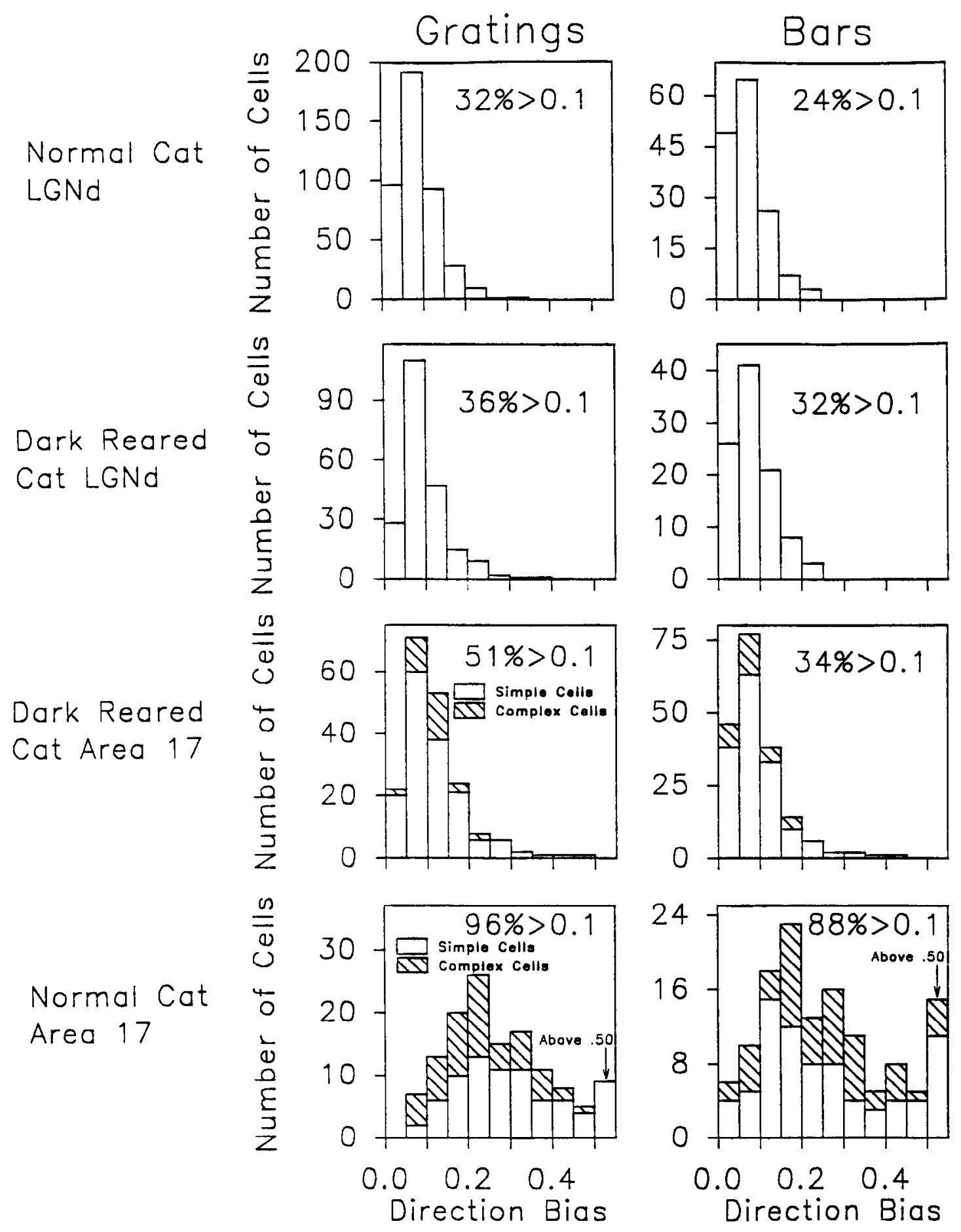

Figure 10. The distributions of the direction biases of cells in area 17 and the LGNd of normal and DR cats. Data collected in response to drifting sinusoidal gratings and drifting bars are shown separately. The best directional biases obtained for each cell were used to construct the histograms. A cell was considered to be significantly orientation or direction biased if the bias was greater than or equal to 0.1 (see Materials and Methods). Notice that gratings were more effective than bars in revealing the cell's selectivity. Also notice that the results for the normal LGNd, DR LGNd, and DR area 17 did not differ. Cells in area 17 of normal cats were far more selective than those in the other groups.

\section{Discussion}

The results of this study confirm previous work and show that dark rearing from birth results in a profound reduction in the number of strongly orientation- and direction-sensitive cells in area 17. Compared to previous studies, this study indicates that more cells in dark-reared area 17 exhibit at least some sensitivity to orientation and direction. We do not view this as inconsistent with earlier work for two reasons. The first is that previous studies employed mostly bars as the test stimuli. This study shows that the orientation and direction sensitivities of area 17 and LGNd cells in DR cats are not easily detectable when moving bars are the test stimuli. The second is that our statistical criteria for determining whether or not a cell exhibits significant 
hias ( $\geq 0.1$ ) corresponds to a maximum to minimum response ratio of about $1.5: 1$. While different groups have employed different criteria for judging cells to be direction selective (reviewed in Orban, 1991), most previous workers would almost certainly classify such cells as unselective. In fact, our experience with more qualitative mapping procedures suggests that a cell must exhibit a bias of 0.2 (response ratios of $2.3: 1$ ) or greater to be subjectively judged as biased. In fact, the majority of DR area 17 cells in this study (Figs. 9, 10) exhibited biases of less than 0.2 .

Despite the profound effects of DR upon area 17, this study indicates that there are no differences between the sensitivities of cells in the LGNd of normal and DR cats. Moreover, the orientation and direction sensitivities of cells in the LGNd and area 17 of DR cats did not differ qualitatively or quantitatively.

There is one difference worth noting, however, in another aspect of the visual responses of cells in the DR LGNd and visual cortex. In particular, DR LGNd cells responded more strongly and reliably to visual stimulation than did normal LGNd cells and DR area 17 cells. In marked contrast, DR area 17 cells responded less strongly to visual stimulation than did normal area 17 cells. A decrease in the peak response of DR cortical cells and an increase in response variability has been reported previously (Leventhal and Hirsch, 1980). This finding combined with the finding that the sensitivities of cells in the LGNd and visual cortex of DR cats do not differ raises the interesting possibility that the LGNd cells may actually be more effective than area 17 cells in signaling orientation and direction in deprived animals. In any event, the increased responsiveness of DR LGNd cells combined with the decreased responsiveness of arca 17 cells in DR cats indicates that information transfer at the geniculocortical synapse and/or other intracortical connections is affected adversely by dark rearing.

\section{Implications for the generation of orientation and direction sensitivity in visual cortex}

If the stimulus selectivities of cells in area 17 and the LGNd were both affected by dark rearing, then little could be said about the generation of the receptive field properties of cells in either area. However, the present finding that the sensitivities of area 17 cells but not LGNd cells are dramatically affected by dark rearing allows certain conclusions to be drawn.

First, since the relay cells that project to area 17 exhibit robust responses and normal orientation and direction biases, it is hard to imagine that the loss of selectivity in area 17 is due to the effects of DR upon the LGNd afferents to the visual cortex. Thus, one can conclude that the pronounced orientation and direction selectivity of normal cortical cells arises as a result of intracortical mechanisms.

Second, there has been some question as to whether the corticogeniculate projection contributes to the orientation sensitivity of LGNd cells (Vidyasagar and Urbas, 1982) or whether LGNd orientation sensitivity is a reflection of the orientation sensitivity of retinal ganglion cells (Soodak et al., 1987; Shou and Leventhal, 1989). The present findings shed light on this issue. In particular, the visual responses and orientation sensitivities of cortical cells are very weak in DR cats, especially in the layers projecting to the LGNd (Leventhal and Hirsch, 1980). Nevertheless, the orientation and direction sensitivities of DR LGNd cells are normal. Since the cells comprising the corticogeniculate projections in DR cats exhibit greally reduced responsiveness and specificity, one can conclude that the sen- sitivity of LGNd cells reflects their retinal inputs. In fact, both retinal ganglion cells and LGNd relay cells are known to be relatively insensitive to visual deprivation when compared to the visual cortex (reviewed in Hirsch and Leventhal, 1978; Movshon and Van Sluyters, 1981; Sherman and Spear, 1982). It should be noted, however, that the strength of surround antagonism of LGNd cells has been suggested to depend upon the orientation sensitivity of cells comprising the corticogeniculate projection (Sillito et al., 1993).

Third, unlike in normal cats, the orientation and direction sensitivities of LGNd and area 17 cells in DR cats are qualitatively and quantitatively similar. In both areas the cells exhibit similar degrees of selectivity and exhibit selectivities that are similarly spatial frequency dependent. Moreover, in both areas bars are less effective than gratings in revealing the selectivities of the cells. These similarities raise at least two possibilities. The first is that dark rearing affects intracortical circuitry and eliminates cortically mediated orientation and direction selectivity. In the absence of normal cortical function, area 17 cells "default" to the properties of their LGNd afferents and thus express selectivities reminiscent of LGNd cells. The second possibility is that the LGNd afferents to area 17 normally provide a weakly orientation- and direction-sensitive substrate which, during development, intracortical mechanisms expand upon and modify. Regardless of which of these possibilities is correct, the present results clearly indicate that the elegant functional architecture of visual cortex (Hubel and Wiesel, 1962) depends in large part upon experience-sensitive, intracortical circuitry.

\section{References}

Albus K, Wolf W, Beckman R (1983) Orientation bias in the response of kitten LGNd neurons to moving light bars. Dev Brain Res 6:308313.

Batschelet E (1981) Circular statistics in biology. New York: Academic.

Blakemore C, Van Sluyters RC (1975) Innate and environmental factors in the development of the kitten's visual cortex. J Physiol (Lond) 248:663-716.

Buisseret P, Imbert M (1976) Visual cortical cells: their developmental properties in normal and dark rear kittens. J Physiol (Lond) 255:511525.

Cleland BG, Levick WR (1974) Brisk and sluggish concentrically organized ganglion cells in the cat's retina. J Physiol (Lond) 162:403431.

Enroth-Cugell C, Robson JG (1966) The contrast sensitivity of retinal ganglion cells of the cat. J Physiol (Lond) 187:517-552.

Fernald R, Chase R (1971) An improved method for plotting retinal ganglion cclls of the cat. J Physiol (Lond) 258:433-452.

Fregnac Y, Imbert M (1978) Early development of visual cortical cells in normal and dark-reared kittens: relationship between orientation selectivity and ocular dominance. J Physiol (Lond) 278:27-44.

Hammond P, Pomfrett CJD (1989) Influence of spatial frequency on tuning and bias for orientation and direction in the cat's striate cortex. Vision Res 30:359-369.

Henry GH (1991) Afferent inputs, receptive field properties and morphological cell types in different laminae of the striate cortex. Neural Basis Vis Function 4:223-240.

Hirsch HVB, Leventhal AG (1978) Functional modification of the developing visual system. In: Handbook of sensory physiology: development of sensory systems (Jacobson M, ed), pp 279-335. New York: Springer.

Hochstcin S, Shaplcy RM (1976) Quantitative analysis of retinal ganglion cell classifications. J Physiol (Lond) 262:237-239.

Hubel DH, Wiesel TN (1962) Receptive fields, binocular interaction and functional architecture in the cat's visual cortex. J Physiol (Lond) 160:106-154.

Imbert M, Buisseret P (1975) Receptive field characteristics and plastic properties of visual cortex cells in kittens reared with or without visual experience. Exp Brain Res 22:25-36. 
Jacobson M (1991) Development neurobiology, 3d ed. New York: Plenum.

Kratz KE, Sherman SM, Kalil R (1979) Lateral geniculate nucleus in dark-reared cats: loss of $Y$ cells without changes in cell size. Science 203:1353-1355.

Leventhal AG, Hirsch HVB (1977) Effects of early experience upon the orientation sensitivity and the binocularity of neurons in the cat visual cortex. Proc Natl Acad Sci USA 74:1271-1276.

Leventhal AG, Hirsch HVB (1980) Receptive-field properties of different classes of neurons in visual cortex of normal and dark-reared cats. J Neurophysiol 43:1111-1132.

Leventhal AG, Schall JD (1983) Structural basis of orientation sensitivity of cat retinal ganglion cells. J Comp Neurol 220:465-475.

Levick WR, Thibos LN (1982) Analysis of orientation bias in cat retina. J Physiol (Lond) 329:243-261.

Mardia KV (1972) Statistics of directional data. New York: Academic.

Movshon JA, Van Sluyters RC (1981) Visual neural development. Annu Rev Psychol 32:477-522.

Orban GA (1991) Quantitative electrophysiology of visual cortical neurones. Neural Basis Vis Function 4:173-217.

Pettigrew JD, Cooper ML, Blasdel GG (1979) Improved use of tapetal reflection for eye-position monitoring. Invest Ophthalmol Vis Sci 18: 490-495.

Sherman SM, Spear PD (1982) Organization of visual pathways in normal and visually deprived cats. Physiol Rev 62:738-855.

Shou T, Leventhal AG (1989) Organized arrangement of orientation sensitive relay cells in the cat's dorsal lateral geniculate nucleus. $J$ Neurosci 9:4287-4302.

Sillito AM, Cudeiro J, Murphy PC (1993) Orientation sensitive elements in the corticifugal influence on centre-surround interactions in the dorsal lateral geniculate nucleus. Exp Brain Res 9:6-16.

Smith EL III, Chino YM, Ridder WR III, Kitagawa K, Langston A
(1990) Orientation bias of neurons in the lateral geniculate nucleus of macaque monkeys. Vis Neurosci 5:525-545.

Soodak RE, Shapley RM, Kaplan E (1987) Linear mechanism of orientation tuning in the retina and lateral geniculate nucleus of the cat. J Neurophysiol 58:267-275.

Stone J, Fukuda Y (1974) Properties of cat retinal ganglion cells: a comparison of W-cells with X-and Y-cells. J Neurophysiol 24:722848.

Thibus LN, Levick WR (1985) Orientation bias of brisk-transient $Y$-cells of the cat retina for drifting and alternating gratings. Exp Brain Res 58:1-10.

Thompson KG, Shou T, Zhou Y, Leventhal AG (1989) Orientation sensitivity of relay cells in the cat lateral geniculate nucleus. Soc Neurosci Abstr 15:175.

Thompson KG, Zhou Y, Leventhal AG (1994a) Direction sensitive $X$ and $Y$ cells within the $A$ laminae of the cat's lateral geniculate nucleus. Vis Neurosci, in press.

Thompson KG, Leventhal AG, Zhou Y, Liu D (1994b) Stimulus dependance of orientation and direction sensitivity of cells in the cat's lateral geniculate nucleus and striate cortex. Vis Ncurosci, in press.

Vidyasagar TR, Urbas JV (1982) Orientation sensitivity of cat LGNd neurones with and without inputs from visual cortical areas 17 and 18. Exp Brain Res 46:157-169.

Wörgötter F, Eysel UT (1987) Quantitative determination of orientational and directional components in the response of visual cortical cells to moving stimuli. Biol Cybern 57:349-355.

Wörgötter F, Grundel O, Eysel UT (1990) Quantification and comparison of cell properties in cat's striate cortex determined by different types of stimuli. Eur J Neurosci 2:928-941.

Zar JH (1974) Circular distributions. In: Biostatistical analysis. Englewood Cliffs, NJ: Prentice-Hall. 